\title{
Polymyxin B: An ode to an old antidote for endotoxic shock
}

\author{
Vikrant M. Bhor, ${ }^{a}$ Celestine J. Thomas, $\dagger^{a}$ Namita Surolia ${ }^{b}$ and Avadhesha Surolia*a \\ Received 18th January 2005, Accepted 6th July 2005 \\ First published as an Advance Article on the web 29th July 2005 \\ DOI: $10.1039 / \mathrm{b500756a}$
}

Endotoxic shock, a syndrome characterized by deranged hemodynamics, coagulation abnormalities, and multiple system organ failure is caused by the release into the circulation of lipopolysaccharide (LPS), the structurally diverse component of Gram-negative bacterial outer membranes, and is responsible for 60\% mortality in humans. Polymyxin B (PMB), a cyclic, cationic peptide antibiotic, neutralizes endotoxin but induces severe side effects in the process. The potent endotoxin neutralizing ability of $\mathrm{PMB}$, however, offers possibilities for designing non-toxic therapeutic agents for combating endotoxicosis. Amongst the numerous approaches for combating endotoxic shock, peptide mediated neutralization of LPS seems to be the most attractive one. The precise mode of binding of PMB to LPS and the structural features involved therein have been elucidated only recently using a variety of biophysical approaches. These suggest that efficient neutralization of endotoxin by PMB is not achieved by mere binding to LPS but requires its sequestration from the membrane. Incorporation of this feature into the design of endotoxin neutralizing peptides should lead to the development of effective antidotes for endotoxic shock.

\section{Introduction}

Though antibiotics acquired prominence in biology and medicine with the ground breaking work of Alexander Fleming on penicillins in the late 1930's, the first demonstrated example of the occurrence of this class of molecules happens to be a polycationic peptide, gramicidin. However, the acute toxicity of gramicidin has prevented its use in clinics. ${ }^{1}$ Polymyxin B (PMB), a cyclic cationic peptide from Bacillus

${ }^{a}$ Molecular Biophysics Unit, Indian Institute of Science, Bangalore 560 012, India.E-mail: surolia@mbu.iisc.ernet.in; Fax: 91-80-2360-0535; Tel: 91-80-2293-2714

${ }^{b}$ Jawaharlal Nehru Centre for Advanced Scientific Research, Bangalore 560064 , India

$\uparrow$ Present address: Howard Hughes Medical Institute, Department of Biochemistry, University of Texas Southwestern Medical Center, 6001 Forest Park Rd, Dallas, TX 75390-8816, USA. polymyxa discovered in 1947, on the other hand has had a mixed fortune in this context. Although it has not been used as extensively as penicillins, it has been used considerably for treating certain bacterial infections such as the meningial infections caused by Haemophilus influenzae, urinary tract infections of Escherichia coli and bacteremia caused by Enterobacter aerogenes etc. and more so for treating a common but often fatal disease called "endotoxic" or "septic" shock, ${ }^{2-4}$ which is caused by "endotoxin" a compound with a fascinating history.

\section{Endotoxin: A historical perspective}

In 1894, the Italian pathologist, Eugenio Centanni extracted endotoxin form Salmonella typhi and other Gram-negative bacteria, calling it 'Pirotoxina' in view of its fever inducing, pyrogenic, properties. In describing endotoxin, Centanni

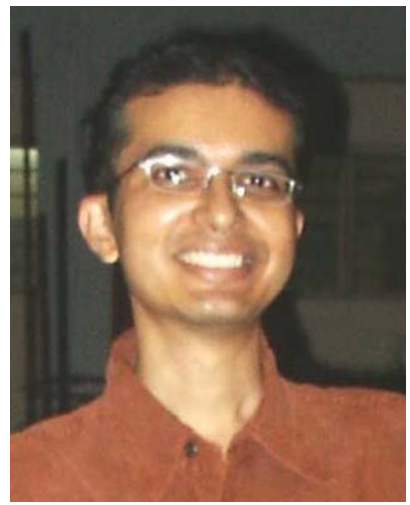

Vikrant M. Bhor
Vikrant M. Bhor is a Research Associate at the Molecular Biophysics Unit, Indian Institute of Science, Bangalore, India. He obtained his PhD from the Department of Life Sciences, University of Mumbai, Mumbai, India, under the supervision of Professor S. Sivakami, in the year 2003. His doctoral work consisted of studies on oxidative stress and alterations in intestinal membrane fluidity during diabetes mellitus.

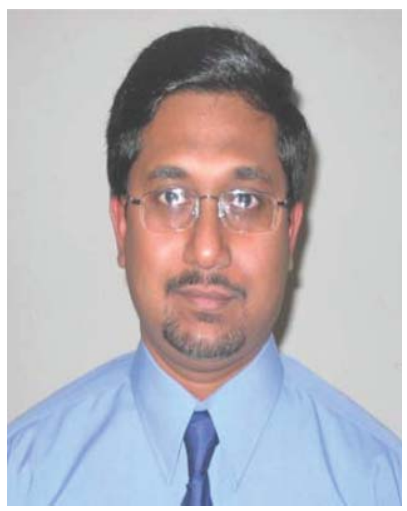

Celestine J. Thomas
Celestine J. Thomas completed his PhD degree at the Molecular Biophysics Unit, Indian Institute of Science, Bangalore, India under the supervision of Professor A. Surolia, in the year 2000. His doctoral work consisted of thermodynamic and kinetic analyses of endotoxin peptidelprotein interactions. He is currently working as a post-doctoral fellow at the Howard Hughes Medical Institute, Department of Biochemistry, University of Texas Southwestern Medical Center, Dallas, Texas. 
stated that "This toxin is ubiquitous to many bacterial genera, it is found in both the pathogenic and non-pathogenic ones, with always the same properties" - a significant observation which was far ahead of its time as borne out by studies early in the next century which established it as an important structural component common to all Gram-negative bacterial outer membranes. ${ }^{5}$ Also in 1894, William Coley in New York described the tumor-necrotizing property of high doses of endotoxin, which, as we know today is due to its potent tumor necrosis factor-inducing properties. ${ }^{6}$ Severe toxicity has precluded the use of endotoxin as an anticancer agent, but this finding which points to its potential beneficial effects has, in recent years, kindled interest in the development of analogs divested of its toxic attributes. In spite of being discovered as early as 1894, the chemical identity of endotoxin as lipopolysaccharide (LPS) was established only in the 1950s and its physical and biological activities were elucidated in the late 1970s. ${ }^{7}$ Uncertainties about the toxic domain of the LPS molecule were resolved two decades ago when chemically synthesized lipid A became available. It was shown to possess all the noxious properties of LPS. ${ }^{8}$

\section{Structural diversity of LPSs}

The LPS molecule comprises three distinct components of contrasting physicochemical properties. These are namely the variable, species-specific, O-polysaccharide region consisting of repeating sugar units, the genera-specific core polysaccharide region and the structurally diverse lipid portion called "Lipid A" (Fig. 1a). The serotype specificity of each bacterial strain is determined by the nature and the number of sugars within a unit, the nature of the linkages of the sugars as well as the number of repetitive units within the O-polysaccharide region. The core polysaccharide region consisiting of 10-12 sugars is comparatively less variable within a genus e.g. the genus Salmonella has a single core structure whereas $E$. coli has five different types. ${ }^{9}$ The proximal portion of the core region consists of heptose residues often substituted by phosphate, pyrophosphate and diphosphoethanolamine (PPEtN) and the distal portion consists of neutral and amino hexoses such as D-glucose, D-galactose, D-glucosamine, D-galactosamine or $N$-acetyl derivatives. The core region is linked to lipid A through an acidic sugar, generally 3-deoxy-D-manno-oct-2ulopyranosonic acid (Kdo). The most well studied lipid A moiety is that found in enteric bacteria (Fig. 1b). The enterobacterial lipid A consists of a $\beta-1,6$ linked diphosphoryl D-glucosamine (D-GlcN) disaccharide with phosphate groups at positions C-1 and C-4' and up to seven hydroxylated fatty acid residues in ester or amide linkage. Additional negatively and positively charged moieties such as PEtN, 4-amino-4deoxy-L-arabinopyranose and D-galacturonic acid, can be present at the anomeric center and at the C-4' hydroxyl. The lipid A disaccharide in certain bacteria is composed of D-2,3diamino-2,3-dideoxyglucose (D-GlcN3N). ${ }^{7,9}$ Variability in lipid A structures brought about by changes in the pattern of substitution of the two lipid A phosphates, the type of fatty acids as well as the degree of acylation contribute to a large extent towards the diversity of LPSs (Fig. 1b). In addition to variations in lipid A structures between different bacterial genera, variations have also been found to occur amongst different species within the same genus e.g. Bordatella, Yersinia and Helicobacter exhibit striking structural differences in lipid A at the species level. ${ }^{9}$

\section{Endotoxic shock}

Endotoxin is an overwhelmingly powerful poison the actions of which target virtually every cell-type in the susceptible animal, and in this way endotoxin evokes a multitude of biological responses. Man is extraordinarily sensitive to endotoxin and miniscule (nanomolar) quantities of it are sufficient to evoke an acute fever response. ${ }^{10,11}$ Exposure to still higher doses, which, in clinical settings usually occurs during systemic bacterial infections, results in a constellation of symptoms termed "endotoxic shock" or "septic shock", a syndrome characterized by deranged hemodynamics,

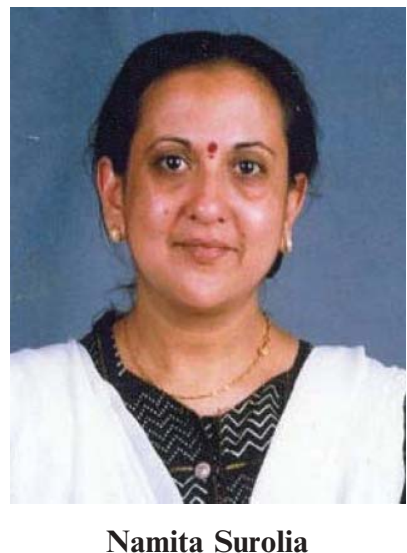

Namita Surolia is an Associate Professor at the Molecular Biology and Genetics Unit, Jawaharlal Nehru Centre for Advanced Scientific Research. Her major contributions are in the field of endotoxic shock and malaria. Her research on the biochemistry of the malarial parasite has led to the discovery of two significant biosynthetic pathways, namely the heme biosynthesis pathway and the fatty acid biosynthesis pathway. Furthermore, her findings of the inhibition of the malarial fatty acid biosynthesis pathway by triclosan have opened up new avenues for the treatment of malaria.

Avadhesha Surolia is Professor at the Molecular Biophysics Unit of the Indian Institute of Science, Bangalore, India. His

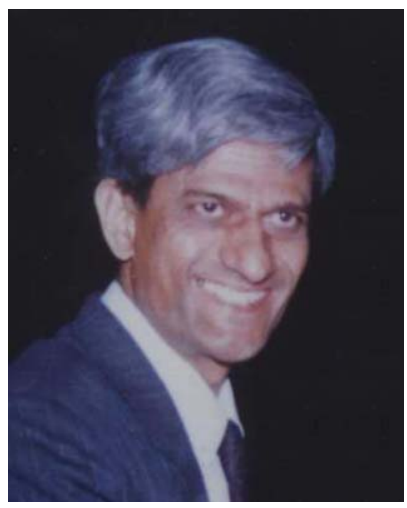

pioneering contributions over the past thirty years have strongly influenced research on structure and function of lectins, orientation and dynamics of cell surface carbohydrate receptors and protein folding, internationally. His original strategies for drug and DNA delivery are widely acclaimed. He explained the enigmatic endotoxin neutralizing activity of polymyxin $B$ to its specific ability to remove endotoxin from its assembly and attributed it to its unique Avadhesha Surolia amphiphilicity. He popularized Protein A as a tool in immuno$\log y$ and discovered the fatty acid synthesis pathway in the human malarial parasite that is distinct from its human host and its interference by triclosan, a commonly used biocide paving the way for novel drug development for treating malaria. 


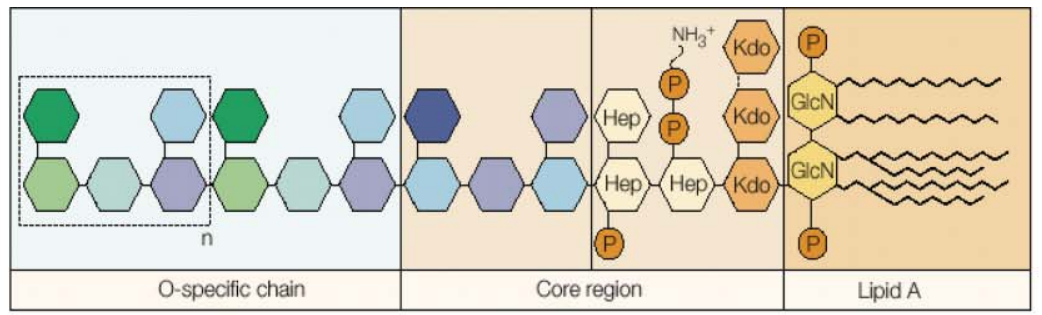

(a)

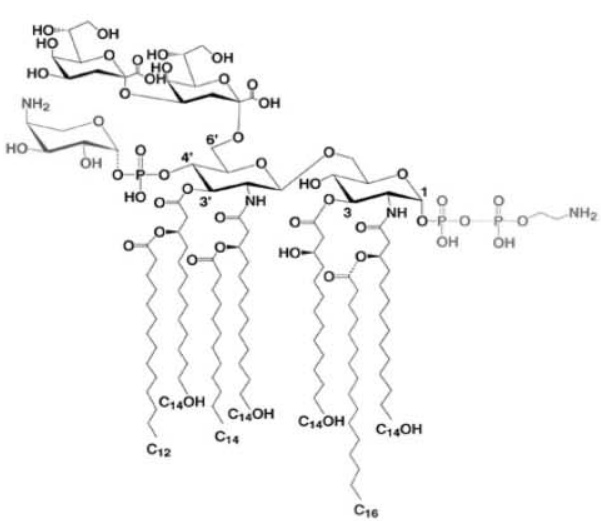

Escherichia coli

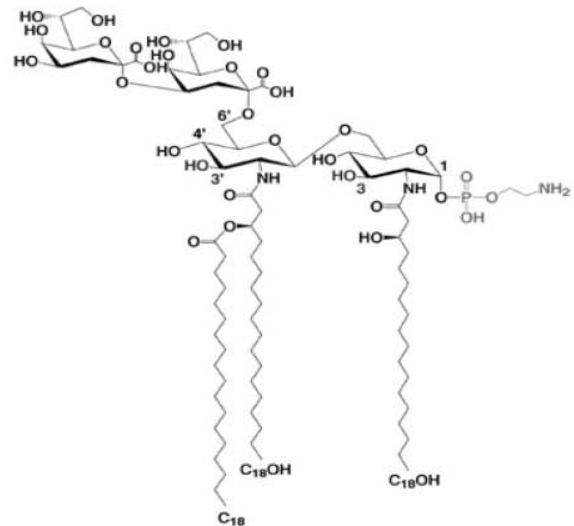

Helicobacter pylori

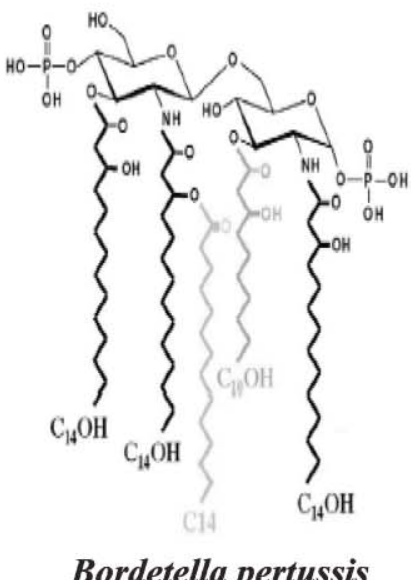

(b)<smiles>[R6][14CH2][14CH2][14CH2][14CH2][14CH2][14CH2][14CH2][14CH2][14CH2][14CH2]</smiles>

Polymyxin B

\section{$\mathrm{H}_{2} \mathrm{~N}-\mathrm{KTKCKFLKKC-COOH}$}

Cyclic decapeptide

\section{$\mathrm{H}_{2} \mathrm{~N}$-CKFLKKC-COOH}

Cyclic heptapeptide

(c)

Fig. 1 Chemical structures of: (a) Lipopolysaccharide comprising of three distinct components of contrasting physicochemical properties. Namely the variable, species-specific, O-polysaccharide region, the genera specific core polysaccharide region and the invariant portion, lipid A. Abbreviations: GlcN, D-glucosamine; Hep, L-glycero-D-mannoheptose; Kdo, 2-keto-3-deoxy-octulosonic acid; P, phosphate. Reprinted from Nat. Rev. Immunol., 3, B. Beutler and E. T. Reitschel, Innate Immune sensing and its roots: the story of endotoxin, 169-176, Copyright (2000), with permission from Nature publishing group (http://www.nature.com/). (b) Structural diversity of lipid A. Bordetella pertussis lipid A structure reprinted from Microbes Infect., 4, M. Caroff, D. Karibian, J. Cavaillon and N. Haeffner-Cavaillon, Structural and functional analysis of bacterial lipopolysaccharides, 915-926, Copyright (2002), with permission from Elsevier. (c) Polymyxin B and its analogs. 


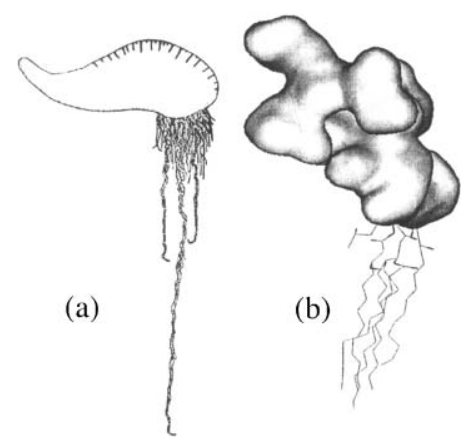

Fig. 2 Schematic representation of the similarity in structural features between the Portuguese man of war and lipopolysaccharide. (a) The Portuguese man of war, Physalia physalis. (b) LPS with its sugar/phosphate head group and acyl chains. Reproduced from C. J. Thomas, PhD Thesis, Indian Institute of Science, Bangalore, India, 2000.

coagulation abnormalities, and multiple system organ failure. ${ }^{12}$ The incidence of endotoxic shock has been rising worldwide largely due to an increase in invasive procedures and, ironically, due to the escalating use of antibiotics; the lysis of bacteria by antibiotics releases the toxin into the systemic circulation precipitating endotoxic shock. Mortality rate in patients with endotoxic shock is about $60 \%$. A priori this fact while disturbing is entirely understandable as current treatments of sepsis are merely supportive and non-specific. ${ }^{13-16}$ The development of specific therapeutics for treating sepsis, therefore, hardly needs any emphasis. Fortunately the possibility of specific therapeutics for sepsis now appears quite feasible, because the mode of action of endotoxin on the target cells is now understood in considerable detail.

\section{Mode of endotoxin action}

At the outset it must be realized that endotoxins per se are not poisonous and quite contrary to other toxins, their insidious nature stems from their ability to excite macrophages and their close kins monocytes, endothelial cells etc., to over-react to their presence. ${ }^{5}$ In this respect, their superficial similarity to the innocent looking but wily Portuguese man of war (Physalia physalis) is amazing (Fig. 2). ${ }^{17}$ In a nutshell the mode of endotoxin action is as follows.

During bacterial death or division, endotoxins are released into the blood stream. Subsequently they get associated with a circulating protein called the lipopolysaccharide binding protein (LBP). It appears that LBP can recognize endotoxins from almost all types of Gram-negative bacteria. This promiscuous alliance between LBP and LPS is responsible for the apparent non-specificity of endotoxins in eliciting the disease irrespective of the bacterial source. This unholy union then goads a receptor, CD-14, on the surface of macrophages and their aforementioned relatives to do their bidding. ${ }^{18-20}$ The complex then recruits a member from the Toll-like receptors (TLRs) family, TLR4, a transmembrane protein with an extracellular domain containing multiple leucine rich repeats (LRR) and an intracellular Toll/interleukin receptor homology domain (TIR) with the aid of a small secreted glycoprotein, myeloid differentiation protein-2 (MD-2) which enhances LPS responsiveness. Spurred by its apparently excitable partners, TLR4 undergoes self-association to form a dimer ${ }^{21}$ and instructs the cellular machinery relentlessly to produce inflammatory cytokines such as tumor necrosis factor, interleukin-1, interleukin-6 and interleukin-8.

TLR4 mediated signaling occurs in two ways, myeloid differentiation factor 88 (MyD88) dependent and MyD88 independent (Fig. 3). Activated TLR4 sequentially recruits the adaptor protein MyD88 and IL-1 receptor associated kinase (IRAK) 1 and 4. IRAK's transiently associate with the receptor complex and upon release associate with and activate tumor necrosis factor (TNF) receptor-associated factor 6 (TRAF6) which in turn activates inhibitors of $\kappa \mathrm{B}$ (IкB) kinase (IKK) complex and mitogen activated protein (MAP) kinase. MAP kinase activates the transcription factor, activator protein-1 (AP-1) through c-Jun N-terminal kinase (JNK) and p38 MAP kinase whereas the IKK complex brings about phosphorylation and subsequent degradation of $\mathrm{I} \kappa \mathrm{B}$ liberating $\mathrm{NF}-\kappa \mathrm{B}$ and leading to generation of inflammatory cytokines. The MyD88 independent activation of NF- $\mathrm{KB}$ and cytokine production is accompanied by phosphorylation and nuclear translocation of IFN- $\beta$ (interferon- $\beta$ ) regulatory factor 3 (IRF3) leading to up-regulation of a set of genes, including IFN- $\beta$ which in turn activates signal transducer and activator of transcription 1 (STAT1) and induction of IFN inducible genes. A TIR containing protein, the TIR-domain containing adaptor inducing IFN- $\beta$ (interferon- $\beta$ ) (TRIF) or the TIR-domain containing molecule (TICAM)-1 is also involved in MyD88 independent pathway. Another TIR domain containing protein called the TIR adaptor protein (TIRAP) or MyD88 like (Mal) protein is also involved in TLR4 mediated signaling but it's exact role remains to be determined. ${ }^{22,23}$

Thus having succumbed to the charm of endotoxin, these macrophages also produce reactive oxygen species such as superoxide anion $\left(\mathrm{O}_{2}{ }^{-}\right)$, hydrogen peroxide $\left(\mathrm{H}_{2} \mathrm{O}_{2}\right)$, nitric oxide (NO) etc. ${ }^{5,24}$ Accentuated production of the inflammatory cytokines and oxygen free radicals by these cells under the influence of endotoxin initially leads to a mild fever, which, in many instances alerts the cells of the immune system to summarily dispose of the invading microorganism and thus help the recovery process. However, if the infection is severe and should the production of inflammatory cytokines and oxygen free radicals by macrophages continue unabated then low blood pressure and unchecked blood clotting throughout the body occurs. Hypotension and disseminated intravascular clotting result in decreased perfusion of vital organs. Ischaemia of vital organs follows causing their failure. $^{25}$ At this stage, the body is in the grip of lethal endotoxic shock.

Numerous studies have indicated that mitochondrial dysfunction and damage could play a role in the pathophysiology of multiple organ failure during prolonged sepsis. Increased generation of nitric oxide and other reactive oxygen species is believed to cause inhibition of the respiratory chain complexes and the resultant fall in ATP levels and tissue oxygen consumption. The consequent reduction in energy availability can lead to a metabolic and physiological breakdown and ultimately manifest in multiple organ failure. ${ }^{26}$ 


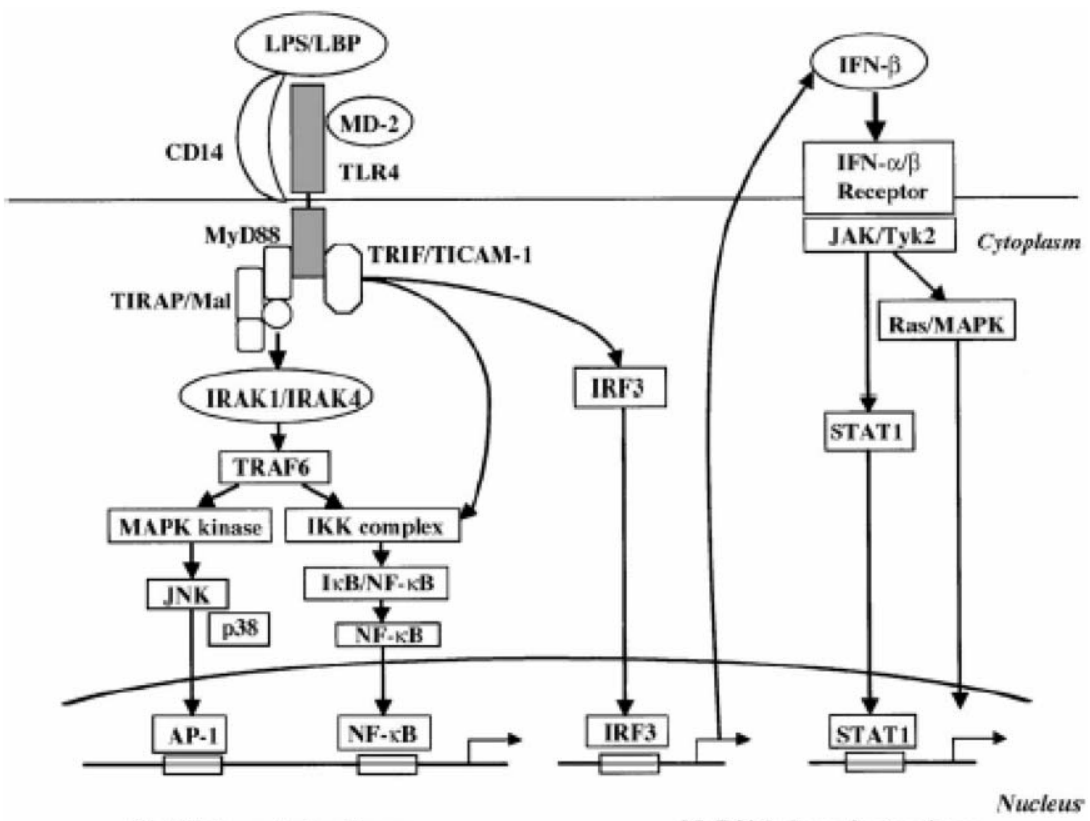

MyD88-dependent pathway

MyDs8-independent pathway

Fig. 3 LPS induced TLR4 mediated signaling (for details see text). Reprinted from Pharmacol.Ther, 100, Fujihara, M., Muroi, M., Tanamoto, K., Suzuki, T., Azuma, H. and Ikeda, H., Molecular mechanisms of macrophage activation and deactivation by lipopolysaccharide roles of the receptor complex, 171-194, Copyright (2003), with permission from Elsevier.

\section{Strategies for combating endotoxic shock}

Our current appreciation of the mode of action of endotoxin has led to the development of several experimental approaches for treating septic shock. These include the inhibition of LPS biosynthesis and release, ${ }^{27,28}$ the sequestration of LPS by peptides or anti LPS antibodies thus preventing its binding to LBP, ${ }^{2,29,30}$ blocking of LPS receptors, ${ }^{31}$ use of antagonistic homologs of $\operatorname{LPS}^{32-35}$ that hinder its shepherding by LBP to the target cells or molecules that abrogate signaling to the pathways leading to the production of inflammatory cytokines such as tumor necrosis factor (TNF), interleukin-1 etc. ${ }^{36,37}$ as well as use of protease inhibitors for preventing the conversion of pro-TNF into active TNF $\alpha .{ }^{38}$ However, in spite of the existence of numerous therapeutic approaches, treatment of endotoxic shock still remains difficult. The fact that septic shock is a complex array of signaling pathways leading to the production of numerous inflammatory mediators that is difficult to curtail once initiated indicates that the blockage of single secondary mediators may hardly alter the overall outcome. Therefore preventing the initiation of the inflammatory cascade using LPS neutralizing agents like polymyxin B may have better value in treatment of endotoxic shock.

\section{Endotoxin and Polymyxin B interaction}

Polymyxin B consists of a cyclic heptapeptide ring formed by an amide bond between the $\gamma$-amino group of diaminobutyric acid (DAB) at position 4 and the carboxyl group of the C-terminal, and a tripeptide tail which is attached to a small fatty acyl chain via a peptide bond. Its amphiphilic character is due to the presence of both a polycationic heptapeptide ring containing five positively charged $\mathrm{DAB}$ residues and a hydrophobic acyl chain (Fig. 1c). Though PMB is known to exert severe side effects such as nephrotoxicity and neurotoxicity, which restrict its utility as a systemic agent for the treatment of sepsis, ${ }^{39}$ its efficient endotoxin neutralizing ability, offers possibilities for designing non-toxic therapeutic agents for combating endotoxicosis. The precise mode of binding of PMB to LPS and the structural features involved therein have been elucidated only recently and in turn should facilitate the achievement of such a goal.

\section{Isothermal titration calorimetric studies}

The tools for studying the interaction between LPS and PMB are limited by the fact that neither of them contains either a strong chromophore or a fluorophore. However, since all biological reactions are accompanied by changes in heat, calorimetry provides an ideal system for probing the nature and magnitude of the forces involved in this interaction. Isothermal titration calorimetry (ITC) is a rapid, accurate and direct method of estimating thermodynamic parameters such as enthalpy $(\Delta H)$, binding constant, stoichiometry and heat capacity change $\left(\Delta C_{\mathrm{p}}\right)$. The results of a typical titration calorimetry experiment for PMB-LPS (E. coli O55 : B5) interaction at $19.4{ }^{\circ} \mathrm{C}$ (Fig. 4) show that PMB binds to LPS stoichiometrically and non-co-operatively with micromolar affinity. This interaction is driven primarily by a favorable change in entropy and is endothermic in nature. An analysis of the results yielded a positive change in the enthalpy $(\Delta H)$ of $39 \mathrm{~kJ} \mathrm{~mol}^{-1}$, an equilibrium association constant $\left(K_{\mathrm{a}}\right)$ of $1.2 \times 10^{6} \mathrm{M}^{-1}$ and a stoichiometry of 1 for PMB bound per mol of LPS. It is pertinent to mention here that the endotoxin-PMB interactions occurred with positive changes in enthalpy and with a stoichiometry of 1 irrespective of the 


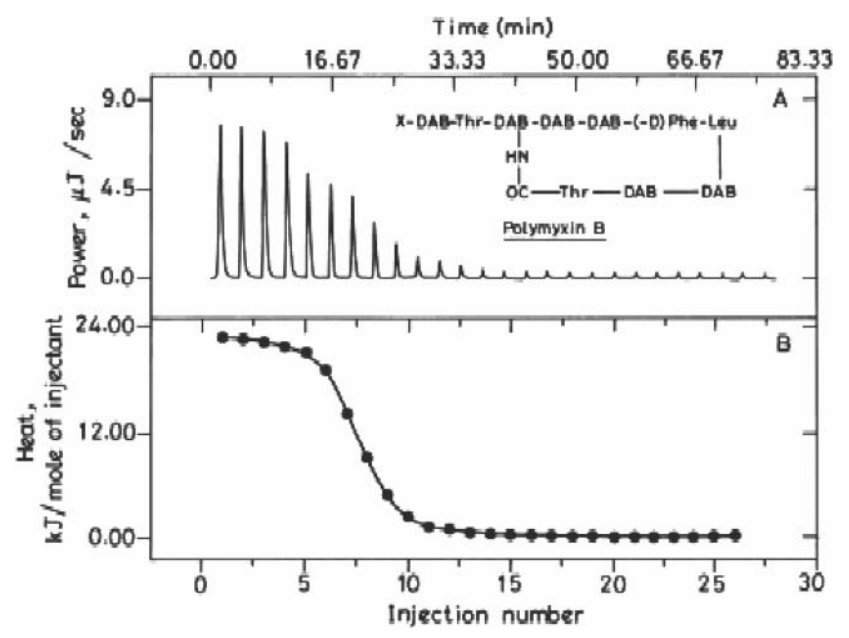

Fig. 4 Isothermal titration calorimetry for the binding of PMB to LPS. Reproduced with permission from Srimal, S., Surolia, N., Balasubramanian, S. and Surolia, A. (1996) Biochem. J., 315, 679$686^{\circledR}$ Biochemical Society.

bacterial source of LPS or the structure of its lipid A moiety or the mode of presentation of endotoxin i.e. in free solution or incorporated in liposomes. The entropy driven nature of the LPS-PMB reaction highlights the dominance of hydrophobic interactions for this reaction. In addition, as expected for a hydrophobically driven reaction, the strength of binding $\left(K_{\mathrm{a}}\right)$ increases while $\Delta H$ decreases with increasing temperature. From the temperature dependence of the change in enthalpy, $\Delta C_{\mathrm{p}}$ values of $-2385 \mathrm{~J} \mathrm{~mol}^{-1} \mathrm{~K}^{-1}$, for this reaction are obtained. The large negative $C_{\mathrm{p}}$ values confirm the primacy of hydrophobic interactions in the recognition of endotoxin by $\mathrm{PMB}^{40}$

Despite the presence of five positive charges on the PMB molecule, any significant contribution of ionic interactions in the binding of PMB to LPS, under the conditions of the experiment, is ruled out by several pieces of evidence. These include the absence of any change in the binding affinities with change in $\mathrm{pH}$ and salt concentration, the observation of positive heat capacity changes as well as the loss of binding with reductively methylated PMB which retains all the positive charges present in native PMB. In contrast electrostatic interactions appear to be essential for binding of PMB to negatively charged lipids. Further, the lack of change in binding affinities with endotoxins from different sources, which contain variable numbers of anionic groups, also lends credibility to the idea that the LPS-PMB binding is mediated primarily through hydrophobic interactions. These data taken together led us to propose that the hydrophobic/van der Waals packing component derived from the aliphatic portion of the DAB side chain might contribute significantly to the thermodynamic properties of PMB-LPS interaction. The positive charge of the $\mathrm{NH}_{2}$ groups of the DAB side chains perhaps prevents the confluence of non-polar amino acid side chains by electrostatic repulsion in addition to providing the necessary polarity, which helps in appropriately orienting PMB on the surface of LPS during the initial stages of recognition. This is further supported by the space filling model of PMB which shows that almost all of the amino groups of the DAB side chains are oriented on one side of the molecule while the side chains of non-polar residues project away on the opposite surface of the molecule and suggests that the segregation of the charged polar groups and non-polar groups on the opposite faces of PMB may be responsible for its observed biological activity. ${ }^{40}$ Further, the involvement of hydrophobic interactions in LPS-PMB binding has been verified later by several other studies. ${ }^{41-43}$

Contrary to the findings of the endothermic nature of the LPS-PMB binding as well as the predominance of hydrophobic interactions in the process, ${ }^{40}$ Brandenburg and co-workers ${ }^{44,45}$ reported that the process is exothermic in nature and inferred that the binding involves electrostatic interactions between the positive charges of PMB and the negatively charged LPS. This discrepancy was resolved by recent studies carried out by the same group, which showed that the binding effects are highly phase dependent. Thus at temperatures below the transition temperature, $T_{\mathrm{c}}$ of the hydrocarbon chains (below $30^{\circ} \mathrm{C}$ ), which correspond to the gel phase of the lipids, a predominantly hydrophobic, entropy driven, endothermic reaction occurs, due to the disruption of the ordered water structure and the cation assembly in the lipid A backbone and adjacent molecules. However, at temperatures above the $T_{\mathrm{c}}$ (above $35^{\circ} \mathrm{C}$ ), corresponding to the liquid crystalline phase, an exothermic reaction occurs mainly due to the strong electrostatic interaction of PMB with the negative charges of LPS. ${ }^{46}$

\section{Fast reaction kinetics}

In order to gain further insight into the mechanism of endotoxin recognition by PMB, fast reaction kinetic studies were carried out using stopped flow spectrofluorometry with a fluorescent derivative of PMB (Dansyl-PMB). The time dependent changes in the fluorescence of dansyl-PMB upon binding to LPS reveal that the process consists of a pair of discrete but consecutive association and dissociation reactions both of which are biphasic. This shows that PMB forms an initial complex (PL) with LPS that is later converted to the final complex $\left(\mathrm{PL}^{*}\right)$. The rate of formation of the initial complex is concentration dependent while the second phase of the reaction is concentration independent, indicating that the interaction essentially follows the scheme below

$$
\mathrm{P}+\mathrm{L} \underset{\mathrm{k}_{-1}}{\stackrel{\mathrm{k}_{1}}{\Leftrightarrow}} \mathrm{PL} \underset{\mathrm{k}_{-2}}{\stackrel{\mathrm{k}_{2}}{\Leftrightarrow}} \mathrm{PL}^{*}
$$

Where $k_{1}, k_{2}, k_{-1}$ and $k_{-2}$ are the two association and dissociation rate constants. The values of these rate constants at $20{ }^{\circ} \mathrm{C}$ are $1.98 \times 10^{5} \mathrm{M}^{-1} \mathrm{~s}^{-1}, 0.341,0.458,0.0571 \mathrm{~s}^{-1}$, respectively. ${ }^{47}$ Values of the overall binding constant match with those obtained by the isothermal titration calorimetry studies. ${ }^{40}$

This points to the presence of a kinetic intermediate structurally and spectroscopically different from the reactants or the final complex (Fig. 5). The initial phase appears to be a bimolecular binding step that is relatively sterically unrestrained such that the amphipathic PMB molecule can bind to the lamellar phase of LPS in several orientations. This is followed by a unimolecular step most likely corresponding to a conformational change caused by the insertion of the 


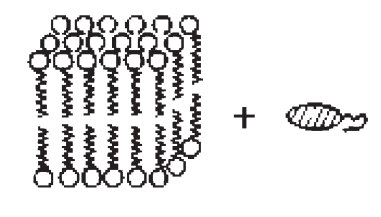

LIPIO
PMB

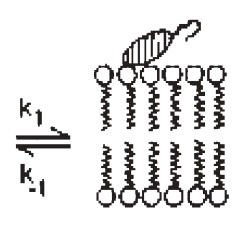

LIPID/PMB

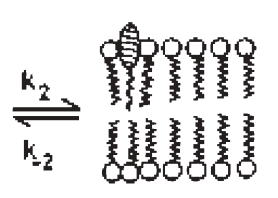

[LIPIO/PMB] *

Fig. 5 Proposed model for the binding of PMB to LPS. Initial binding of PMB to the lamellar phase of LPS leads to the formation of the intermediate PMB-LPS complex. This complex subsequently isomerizes to yield the final complex, PMB-LPS*, which involves the insertion of the acyl chain of PMB into the LPS lamellar phase. Reprinted with permission from Thomas, C. J., Gangadhar, B. P., Surolia, N. and Surolia, A. (1998) J. Am. Chem. Soc., 120, 12428-12434. Copyright (1998) American Chemical Society.

hydrophobic portion of PMB into the non-polar interior of the LPS lamellar phase and entails a large expenditure of energy. ${ }^{47}$

Based on this as well as the inference of the importance of the amphiphilic distribution of amino acid side chains of PMB in the recognition and binding of LPS, peptide mimics of PMB with heightened asymmetric distribution of the hydrophobic and positively charged residues were designed and synthesized. These bound to endotoxin reasonably well. ${ }^{48}$ Unfortunately, however, they were ineffective in protecting mice treated with endotoxin (C. J. Thomas and A. Surolia, Unpublished observations). Though the lack of protective effect could also be attributed to other factors such as degradation and poor bioavailability of the peptide mimics it is certain that binding ability alone appears to be a deceptive indicator of endotoxin neutralization and therefore PMB must possess some unique properties that allow it to neutralize endotoxin effectively. It, thus, became necessary to identify additional physical parameters of the interaction, which may adequately describe the outcome of the recognition of LPS on its biologic activity, as they may aid in the design and screening of molecules with anti-endotoxic activity. This mystery could eventually be solved through studies involving surface plasmon resonance (SPR). ${ }^{49}$

\section{Surface plasmon resonance studies}

SPR is a rapid method for evaluating the elementary steps as well as the binding affinities involved in the interaction between a macromolecule and its complementary ligand. Since study of macromolecule-ligand interaction by SPR depends solely on the mass changes, it can be monitored in real time without taking recourse to any external labels. Moreover, it is perhaps the only technique that can provide data for both the association and dissociation phases of a reaction in a single run. Additionally, in SPR the ability to form model membrane assemblies; monolayers or bilayers, incorporating the biological receptors that mimic the natural environment offer opportunities to study, in molecular terms, the surface associated phenomena.

A time and concentration dependent reduction in the response units (RUs) was observed in the binding of PMB with the lipid $\mathrm{A} / \mathrm{L}-\alpha$-phosphatidylcholine, dimyristoyl (DMPC) monolayer. The time dependent drop in RUs indicates that PMB is probably able to "take off" some mass from such monolayers (Fig. 6). The interaction of PMB with the phospholipid and its sequestration from the surface was ruled

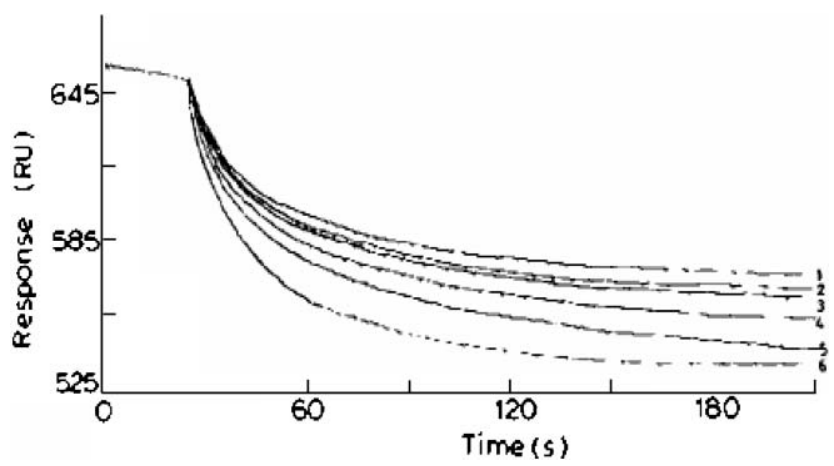

Fig. 6 Surface Plasmon Resonance analysis of the binding of PMB to LPS. The figure depicts the "take off" of the lipid A from lipid A/DMPC monolayers from the chip by PMB as a function of lipid A concentration (curves $1-4$ for 2,5,10 and $25 \mathrm{~mol} \%$ lipid A respectively) and PMB concentration (curves 5 and 6, 125 and $300 \mathrm{nM}$ of PMB respectively). Abbreviation: DMPC, L- $\alpha$-phosphatidylcholine, dimyristoyl. Reproduced from C. J. Thomas, N. Surolia and A. Surolia, J. Biol. Chem., 1999, 274, 29624-29627.

out with the absence of change in RUs in experiments with monolayers made with DMPC alone. On the contrary, a time and PMB concentration-dependent drop in RUs from neat lipid A monolayers was also observed. These findings therefore prove beyond doubt that PMB is able to form a specific complex with endotoxin and sequester it. ${ }^{49}$

\section{Peptide mediated endotoxin neutralization}

Amongst all the possible interventional strategies for combating endotoxic shock, the sequestration and neutralization of circulating LPS by synthetic anti-LPS peptides seems to be the most attractive one. The structural complexity of anti-LPS peptides and their mode of endotoxin recognition rule out development of resistance by susceptible bacteria through mutations or genetic recombination. Another feature that makes them desirable is their widespread presence in a variety of organisms from invertebrates to mammals, hinting at their importance in warding off microbial assault. ${ }^{50,51}$ Further, the possibility of developing peptide mimics resistant to protease degradation in order to prolong their half-life in vivo ${ }^{52}$ adds to their utility as therapeutic agents.

Numerous attempts towards the synthesis of anti-LPS peptides with potent endotoxin neutralizing ability without the PMB associated toxicity have been made. The majority of these peptides were derived from a variety of naturally 
occurring LPS-binding proteins such as serum amyloid P component (SAP) ${ }^{52}$ human neutrophil bactericidal/ permeability-increasing protein (BPI), ${ }^{53}$ Limulus anti-LPS factor (LALF), ${ }^{54}$ lactoferrin, ${ }^{55}$ microbicidal proteins found in azurophilic granules of neutrophils - cationic protein 18 $(\mathrm{CAP} 18)^{56}$ and cationic protein 37 (CAP37), ${ }^{57}$ histones $^{43}$ as well as natural host defense peptides such as magainins ${ }^{58}$ cecropins $^{59}$ and Bac7. ${ }^{60}$

A number of the LPS-binding proteins and peptides such as SAP, lactoferrin, BPI, CAP18 and CAP37 also bind to heparin. The heparin-binding regions of these peptides may or may not correspond to the heparin binding consensus sequence. Though it is not known whether the LPS- and heparin-binding regions overlap, the heparin binding consensus sequence, which consists of basic and hydrophobic regions bears resemblance to the cationic/hydrophobic motifs of LPS-binding molecules. ${ }^{61}$ Recently a number of heparin binding peptides have been shown to possess antimicrobial properties. ${ }^{62}$ This suggests the possibility of using naturally occurring heparin-binding peptides or their analogs for neutralization of LPS.

Besides the use of peptides derived from LPS-binding proteins and peptides, several efforts have been directed towards the development of PMB analogs. ${ }^{42,48,49}$ The efforts at peptide-based endotoxin neutralization have been recently reviewed $^{63}$ and hence a mention of only some of the notable attempts is made here.

\section{LPS binding protein fragments}

SAP-derived peptides, pep27-39, pep61-75 and pep186-200 were found to effectively inhibit both LPS binding and the LPS-induced responses in phagocytes. Carboxyamidomethylation of pep27-39 resulted in enhanced activity whereas pep186-200 was able to prolong the survival of actinomycinsensitized, LPS-injected mice to a limited extent. The charge, hydrophobicity, size as well as amino acid sequence of the peptides were suggested to influence their activity. ${ }^{52,61}$

Beta sheet-forming, BPI-derived 33mer peptides were found to possess both microbicidal and LPS neutralizing activity and charge and hydrophobicity were shown to influence this activity. ${ }^{53}$ Another BPI-derived synthetic peptide, BNEP (BPI 148-161), bound to LPS with an affinity similar to that of PMB and protected LPS-treated animals by decreasing plasma endotoxin and pro-inflammatory cytokines. ${ }^{64}$

A lactoferrin derived synthetic peptide consisting of the $N$-terminal 33 residues of the protein exhibited endotoxin neutralizing ability comparable to PMB and protected animals against lethal endotoxin challenge in contrast to a $27 \mathrm{mer}$ peptide which lacked the initial six basic residues indicating the importance of a cationic head group in neutralizing endotoxin. ${ }^{55}$ The importance of hydrophobicity in the neutralization of LPS was suggested by enhancement of activity of a lactoferrin-derived peptide upon acylation most likely due to a combination of neutralization of negative charges of LPS by the peptide portion as well as the penetration of the hydrophobic core of LPS aggregates by the acyl chain. ${ }^{65}$

Cyclic, cationic peptides based on LALF inhibited LPSinduced responses due to compensation of negative charges of LPS as well as fluidization of lamellar LPS aggregates ${ }^{66}$ Recently histones have been demonstrated to possess potent endotoxin binding properties. All the histone molecules with the exception of $\mathrm{H} 4$ possessed binding affinities higher than PMB and studies with synthetic peptides derived from these proteins indicate that the LPS binding site is located at the $\mathrm{C}$ terminus. However these peptides representing partial structures exhibited affinities much lower than those of the intact proteins. ${ }^{43}$

\section{Host defense peptides}

Bac7(1-35), a synthetic peptide derived from a proline-rich antibacterial peptide from bovine neutrophils, inhibited LPS procoagulant activity and significantly reduced plasma endotoxin levels and lethality rates in endotoxin-challenged rats as effectively as PMB. ${ }^{60}$ Novel analogs of the naturally-occurring antibacterial peptide, magainin, with improved amphiphilicity and ability to adopt helical conformations in membranes were synthesized and their activities evaluated. Three of the peptides exhibited affinities for LPS close to that of PMB. Biophysical characterization of these peptides revealed the importance of electrostatic interactions in the initial binding and hydrophobic interactions in the membrane disruption activity. ${ }^{58}$

\section{Phage displayed peptides}

A fairly different approach for obtaining endotoxin-neutralizing peptides was adopted by Thomas et al. ${ }^{67}$ The technique of phage display by biopanning of a repertoire of a random pentadecapeptide library to select lipid A binding peptides was exploited in these studies. A comparison of the sequence of the 12 peptides so obtained revealed no consensus indicating that the lipid A binding motif is not sequence-specific in accordance with the sequence variation seen in the naturally-occurring endotoxin binding proteins and peptides. The flexibility of the peptides coupled with their plasticity in recognizing lipid A explains their tight binding to endotoxin. Structurally, the asymmetric distribution of the charged polar residues on one face of the helix and non-polar residues on the opposite face appears to correlate with their activity.

\section{PMB conjugates}

PMX622, a covalent conjugate of PMB and Dextran-70, has been designed to combine the endotoxin neutralizing ability of PMB with the favorable colloidal, pharmacokinetic, and metabolic properties of Dextran-70. It was found to effectively neutralize endotoxin in a number of animal models as well as in phase I clinical models and to be significantly less toxic than PMB. However, it was protective only when injected prior to endotoxin challenge. ${ }^{68}$

\section{PMB analogs}

Additionally, as mentioned earlier several PMB analogs namely a cyclic heptapeptide, a cyclic decapeptide (Fig. 1c), a BPI derived 28mer, a 23 mer and a disulfide bond containing cyclic decapeptide have been synthesized and a comparison of their interactions with LPS with those of PMB and PMBN 
with LPS was performed by SPR. None of these peptides exhibited the PMB-like time- and concentration-dependent diminution in RUs, thereby indicating a lack of the ability to sequester endotoxin.

Polymyxin B nanopeptide (PMBN), a proteolytically generated derivative of $\mathrm{PMB}$, which lacks the hydrophobic tail and is a poor antimicrobial compound, possesses a lower amphiphilicity and is able to associate only at the interfacial regions of the LPS lamellar phase, while PMB subsequent to such an interaction is able to penetrate the lamellar assembly primarily through its hydrophobic region. This difference alone appears to be responsible for the poor anti-endotoxic activity of PMBN. The binding of a cyclic decapeptide to LPS was comparable to that of PMB since its amphiphilicity is higher than that of PMBN whereas the cyclic heptapeptide, which displays no amphiphilicity exhibits extremely poor binding to LPS. This further emphasizes the point that amphiphilicity is both necessary and sufficient for binding of peptides to LPS. All the other peptides like PMBN lack the ability to penetrate into the LPS lamellar phase and are therefore of limited therapeutic value. ${ }^{48,49}$

\section{Conclusion}

Thus, in spite of the large body of work on endotoxin neutralizing peptides, the availability of a non-toxic, effective, antidote for endotoxic shock still remains elusive. Studies on the interaction of endotoxin with the cyclic, cationic peptide, PMB using a variety of biophysical approaches clearly demonstrate that effective endotoxin neutralization requires sequestration of LPS from the membrane. The majority of the existing anti-LPS peptides lack this important property and hence are unlikely to replace $\mathrm{PMB}$ in clinical settings. Thus this ode to PMB, an old antidote for endotoxic shock, emphasizes the need to incorporate the ability to solubilize away endotoxin specifically in the design of effective therapeutics.

\section{Acknowledgements}

The authors thank Ranbaxy Research Labs for supporting some of the studies described in this review.

\section{References}

1 R. E. Hancock, Lancet Infect Dis., 2001, 1, 156-164.

2 D. C. Morrison and D. M. Jacobs, Immunocytochemistry, 1976, 13, 813-818.

3 M. Schindler and M. J. Osborn, Biochemistry, 1979, 18, 4425-4430.

4 M. Schindler and M. Teuber, Antimicrob. Agents Chemother., $1975,8,95-104$.

5 B. Beutler and E. T. Reitschel, Nat. Rev. Immunol., 2003, 3, 169-176.

6 H. Coley-Nauts, W. B. Swift and B. L. Coley, Cancer Res., 1946, 6, 205-216.

7 R. H. Raetz and C. Whitfield, Annu. Rev. Biochem., 2002, 71, 635-700.

8 C. Galanos, Eur. J. Biochem., 1985, 148, 1-5.

9 M. Caroff, D. Karibian, J. M. Cavaillon and N. HaeffnerCavaillon, Microbes Infect., 2002, 4, 915-926.

10 L. Leive, Ann. N. Y. Acad. Sci., 1974, 235, 109-129.

11 C. Galanos, O. Luderrzitz, E. T. Reitschel and O. Westphal, Int. Rev. Biochem., 1977, 14, 239-334.
12 P. F. Fink, 1990, in Handbook of Critical Care, ed. J. L. Berk and J. E. Sampliner, Little, Brown and Co., Boston, MA, 3rd edn., p. 619.

13 E. J. Zeigler, N. Eng. J. Med., 1991, 324, 429-436.

14 S. Aldrige, Trends Biotechnol., 1995, 11, 373-375.

15 R. Sundaresan and J. N. Sheagren, Complication. Surg., 1994, 14, 261-268.

16 A. S. Cross and S. Opal, J. Endotoxin. Res., 1994, 1, 57-69.

17 C. J. Thomas, PhD Thesis, Indian Institute of Science, Bangalore, India, 2000.

18 S. E. Goldblum, T. W. Brann, X. Ding, J. Pugin and P. S. Tobais, J. Clin. Infect., 1994, 93, 692-702.

19 E. Hailman, H. S. Lichenstein, M. M. Wulfer, D. S. Miller, D. S. Johnson, M. Kelly, L. A. Busse, M. M. Zukokwski and S. D. Wright, J. Exp. Med., 1994, 179, 269-277.

20 J. A. Gegner, R. A. Ulevitch and P. S. Tobais, J. Biol. Chem., 1995, 270, 5320-5325.

21 H. K. Lee, S. Dunzendorfer and P. S. Tobais, J. Biol. Chem., 2004, 279, 10564-10574.

22 M. Fujihara, M. Muroi, K. Tanamoto, T. Suzuki, H. Azuma and H. Ikeda, Pharmacol. Ther., 2003, 100, 171-194.

23 M. M. Monick and G. W. Hunninghake, Eur. Respir. J., 2002, 20, 210-222.

24 B. Beutler, Curr. Opin. Immunol., 2000, 12, 20-6.

25 B. Beutler, Annu. Rev. Pharmacol. Toxicol., 2003, 43, 609-628.

26 M. Singer, The Biochemist, 2005, 27, 20-22.

27 R. Goldman, W. Kohlbrenner, P. Lartey and A. Pernet, Nature, 1987, 329, 162-164.

28 H. R. Onishi, B. A. Pelak, L. S. Gerckens, L. L. Silver, F. M. Kahan, M. H. Chen, A. A. Patchett, S. M. Galloway, S. A. Hyland, M. S. Anderson and C. R. Raetz, Science, 1996, 274, 980-982.

29 P. Elsbach and J. Weiss, Curr. Opin. Immunol., 1993, 5, 103-107.

30 A. Novogrodsky, A. Vanichken, M. Patya, A. Gazit, N. Osherov and A. Levitzky, Science, 1994, 264, 1319-1322.

31 P. S. Tobais, J. Mathison and R. J. Ulevitch, J. Biol. Chem., 1988, 263, 13479-13480.

32 W. J. Christ, Science, 1995, 268, 80-83.

33 M. Mullarkey, J. R. Rose, J. Bristol, T. Kawata, A. Kimura, S. Kobayashi, M. Przetak, J. Chow, F. Gusovsky, W. J. Christ and D. P. Rossignol, J Pharmacol. Exp. Ther., 2003, 304, 1093-1102.

34 A. V. Demchenko, M. A. Wolfert, B. Santhanam, J. N. Moore and G. J. Boons, J. Am. Chem. Soc., 2003, 125, 6103-6112.

35 B. Santhanam, M. A. Wolfert, J. N. Moore and G. J. Boons, Chemistry, 2004, 10, 4798-4807.

36 A. Xagorari, C. Roussos and A. Papapetropoulos, Br. J. Pharmacol., 2002, 136, 1058-1064.

37 M. Leon-Ponte, M. G. Kirchhof, T. Sun, T. Stephens, B. Singh, S. Sandhu and J. Madrenas, Immunol. Lett., 2005, 96, 73-83.

38 M. A. Palladino, F. R. Bahjat, E. A. Theodorakis and L. L. Moldawer, Nat. Rev. Drug Disc., 2003, 2, 736-746.

39 R. L. Danner, K. A. Joiner, M. Rubin, W. H. Patterson, N. Johnson, K. M. Ayers and J. E. Parrillo, Antimicrob. Agents Chemother., 1989, 33, 1428-1434.

40 S. Srimal, N. Surolia, S. Balasubramanian and A. Surolia, Biochem. J., 1996, 315, 679-686.

41 D. Lorinczy and B. Kocsis, Thermochim. Acta, 2001, 372, 19-23.

42 H. Tsubery, I. Ofek, S. Cohen, M. Eisenstein and M. Fridkin, Mol. Pharmacol., 2002, 62, 1036-1042.

43 L. A. Augusto, P. Decottignies, M. Synguelakis, M. Nicaise, P. L. Marechal and R. Chaby, Biochemistry, 2003, 42, 3929-3938.

44 K. Brandenburg, I. Moriyon, M. D. Arraiza, G. Lewark-Yvetot, M. H. J. Koch and U. Seydel, Thermochim. Acta, 2002, 382, 189-198.

45 K. Brandenburg, M. D. Arraiza, G. Lehwark-Ivetot, I. Moriyon and U. Zahringer, Thermochim. Acta, 2002, 394, 53-61.

46 K. Brandenburg, A. David, J. Howe, M. H. Koch, J. Andra and P. Garidel, Biophys. J., 2005, 88, 1845-1858.

47 C. J. Thomas, B. P. Gangadhar, N. Surolia and A. Surolia, J. Am. Chem. Soc., 1998, 120, 12428-12434.

48 C. J. Thomas and A. Surolia, FEBS Lett., 1999, 445, 420-424.

49 C. J. Thomas, N. Surolia and A. Surolia, J. Biol. Chem., 1999, 274, 29624-29627.

50 M. Zasloff, Proc. Natl. Acad. Sci. U. S. A., 1987, 84, 5449-5453.

51 R. Chaby, Drug Discov. Today, 1999, 4, 209-222. 
52 C. J. C. de Haas, M. E. van der Tol, K. P. Van Kessel, J. Verhoef and J. A. Van Strip, J. Immunol., 1998, 161, $3607-3615$.

53 K. H. Mayo, J. Haseman, E. Ilyina and B. Gray, Biochim. Biophys. Acta, 1998, 1425, 81-92.

54 C. Reid, J. Biol. Chem., 1996, 271, 28120-28127.

55 G. H. Zhang, D. M. Mann and C. M. Tsai, Infect. Immun., 1999, 67, 1353-1358

56 J. W. Larrick, M. Hirata, R. F. Balint, J. Lee, J. Zhong and S. C. Wright, Infect. Immun., 1995, 63, 1291-1297.

57 H. A. Periera, I. Erdem, J. Pohl and J. K. Spitznagel, Proc. Natl. Acad. Sci. U. S. A., 1993, 90, 4733-4737.

58 C. J. Thomas, N. Surolia and A. Surolia, J. Biol. Chem., 2001, 276, 35701-35706.

59 D. Y. Oh Kim, S. Y. Shin, J. H. Kang, K. S. Hahm and K. L. Kim, J. Pep. Res., 1999, 53, 578-589.

60 R. Ghiselli, A. Giacometti, O. Cirioni, R. Circo, F. Mocchegiani, B. Skerlavaj, G. D'Amato, G. Scalise, M. Zanetti and V. Saba, Shock, 2003, 19, 577-581.
61 C. J. C. de Haas, R. van der Zee, B. Benaissa-Trouw, K. P. M van Kessel, J. Verhoef and J. A. Van Strip, Infect. Immun., 1999, 67, 2790-2796.

62 E. Andersson, V. Rydengard, A. Sonesson, M. Morgelin, L. Bjorck and A. Schmidtchen, Eur. J. Biochem., 2004, 271, 1219-1226.

63 M. Jerala and M. Porro, Curr. Top. Med. Chem., 2004, 4, $1173-1184$.

64 Z. Jiang, Z. Hong, W. Xiaoyun, G. Guo, L. Gengfa, L. Yongning and X. Guangxia, Int. Immunopharmacol., 2004, 4, 527-537.

65 J. Andra, K. Lohner, S. E. Blondelle, R. Jerala, I. Moriyon, M. H Koch, P. Garidel and K. Brandenburg, Biochem. J., 2005, 385, 135-143.

66 J. Andra, M. Lamata, G. Martinez de Tejada, R. Bartels, M. H. J. Koch and K. Brandenburg, Biochem. Pharmacol., 2004, 68, 1297-1307.

67 C. J. Thomas, S. Sharma, G. Kumar, S. S. Visweswariah and A. Surolia, Biochem. Biophys. Res. Commun., 2003, 307, 133-138.

68 P. Lake, J. DeLeo, F. Cerasoli, L. Logdberg, M. Weetall and D. Handley, Antimicrob. Agents Chemother., 2004, 48, 2987-2992. 